\title{
SEROPREVALENCIA DE LA CISTICERCOSIS PORCINA EN TRES CASERÍOS DE LA PROVINCIA DE ANDAHUAYLAS, APURÍMAC
}

\author{
Viterbo Ayvar P. ${ }^{1}$, Armando González Z. ${ }^{2,3}$, Néstor Falcón P. ${ }^{2}$, Teresa Bernal R. ${ }^{2}$ y \\ Carla Mena A. ${ }^{1}$
}

\section{Abstract}

\begin{abstract}
Porcine cysticercosis is endemic in rural communities of Peru, due to prevaling conditions contributing to the occurrence, transmission and maintenance of the disease. This paper assessed the real prevalence of porcine cysticercosis in three villages of Andahuaylas province, Apurímac. All pigs in the area were sampled with the exception of suckling piglets and advanced pregnant sows $(n=304)$. The samples were analyzed by the Enzyme Immuno Electro Transfer Blot (EITB) test. Resulting data was evaluated to determine the risk factors and a stocastic simulation was used to asses the real prevalence, using functions that generate random numbers that followed beta binomial distributions. The level of infection in Nueva Esperanza was significantly lower to those found in Turpo and Matapuquio ( $\mathrm{p}<0.05)$. No statistical differences were found due to sex. There was a perfect direct correlation between age and the prevalence of porcine cysticercosis $\left(r_{s}=1\right)$. The logistic regression showed that location and age represented risk factors asociated with the disease $(\mathrm{p}<0.0001)$. Serologic results indicated that the general prevalence was $47.3 \pm 5.6 \%$, while prevalence for Nueva Esperanza was $26.5 \pm 7.6$, for Turpo was $72.3 \pm 9.5$, and for Matapuquio was $54.0 \pm 10.5 \%$. The real prevalence for all three villages would be of $23 \%$, whereas $96 \%$ of the observations were between 17 and $29 \%$. It is concluded that porcine cysticercosis in Andahuaylas villages is a public health problem.
\end{abstract}

Key words: cysticercosis, prevalence, real prevalence, stocastic simulation

\section{RESUMien}

La cisticercosis porcina es endémica en comunidades rurales del Perú debido a la existencia de factores que favorecen la presencia, transmisión y mantenimiento de la enfermedad. El presente trabajo evaluó la prevalencia real de la cisticercosis porcina en tres caseríos (Nueva Esperanza, Turpo y Matapuquio) de la provincia de Andahuaylas, Apurímac. Se muestreó a la totalidad de la población porcina $(n=304)$, exceptuando animales menores de 2 meses y hembras en gestación avanzada. Los sueros se analizaron mediante la prueba de Electro Inmuno Transferencia Blot (EITB). Los datos se analizaron para determinar factores de riesgo y se emplearon en el entorno de una simulación estocástica para estimar la prevalencia real usando funciones de generación de números aleatorios que seguían distribuciones beta-binomiales. El nivel de infección en Nueva Esperanza fue significativamente menor a los encontrados en Turpo y Matapuquio $(\mathrm{p}<0.05)$. No hubo diferencias entre sexos, pero se encontró una correlación directa perfecta $\left(r_{s}=1\right)$ entre la edad de los animales y la prevalencia de cisticercosis. El análisis de regresión logística mostró que las variables zona de muestreo y edad representaron

\footnotetext{
${ }^{1}$ Práctica privada

${ }^{2}$ Laboratorio de Medicina Veterinaria Preventiva, FMV-UNMSM

${ }^{3}$ E-mail: agonzalesz@vet.unmsm.edu.pe
} 
factores de riesgo asociados a la enfermedad $(\mathrm{p}<0.0001)$. Los resultados serológicos indicaron que el porcentaje de prevalencia general fue de $47.3 \pm 5.6 \%$ mientras que las prevalencias para Nueva Esperanza, Turpo y Matapuquio fueron de 26.5, 72.3 y 54.0\%, respectivamente. La prevalencia real para los tres caseríos fue del $23 \%$, encontrándose el 96\% de las observaciones entre el 17 al 29\%. Se concluye que la cisticercosis porcina en los caseríos estudiados es un problema de salud pública.

Palabras clave: cisticercosis, prevalencia, prevalencia real, simulación estocástica

\section{INTRODUCCIÓN}

La cisticercosis porcina constituye un problema de salud pública común en países en vías de desarrollo (Acha y Szyfres, 1992; Correa et al., 1991; OPS, 1994). Las condiciones socioeconómicas, de salubridad y de educación sanitaria existentes en estos países favorecen la permanencia y transmisión de la Taenia solium (Evans et al., 2000; García et al., 1995).

La crianza de porcinos es una actividad de importancia económica para los pobladores andinos. Por ser una crianza extensiva o al pastoreo, permite una alimentación de bajo costo, de manera que el cerdo es utilizado como fuente de ahorro (García et al., 1991; González et al., 1990). Sin embargo, la infección de los cerdos trae consigo importantes pérdidas para la precaria economía del campesino debido a la merma en precio de la carne infectada o al decomiso total (The Cisticercosis working Group in Perú, 1993).

La prevalencia en porcinos es considerada un importante indicador epidemiológico de la infección con $T$. solium en humanos (García et al., 1999b), en donde las elevadas seroprevalencias encontradas en la mayoría de los estudios reflejan la real magnitud de la cisticercosis en nuestro medio (García et al., 1999a). La variación del comportamiento de la enfermedad en las comunidades y la estrecha relación entre la seroprevalencia observada en la población porcina y humana, indica la necesidad de individualizar los enfoques de estrategias de control a corto plazo, para adecuarlas a cada realidad local (García et al., 1999a).
En el presente estudio epidemiológico de diagnóstico serológico de cisticercosis porcina se determinó la prevalencia real de la cisticercosis porcina, usando la prueba de Electroinmuno Transferencia Blot (EITB). Estos datos epidemiológicos permitirán contribuir al conocimiento de la real magnitud del problema, así como la elaboración de mapas ayudarán a clarificar la distribución en la enfermedad y así poder desarrollar estrategias de prevención y control, además de determinar prioridades de intervención.

\section{Materiales y Métodos}

\section{Lugar de estudio y animales}

El estudio se realizó en los caseríos de Nueva Esperanza, Matapuquio y Turpo, localizados en la provincia de Andahuaylas, departamento de Apurímac. Estos caseríos se encuentran en altitudes superiores a los $3,000 \mathrm{msnm}$, ubicados entre 20 y $45 \mathrm{~km}$ de la ciudad de Andahuaylas, capital de la provincia, y con acceso a través de una carretera afirmada y trochas carrozables.

La economía de estos poblados se apoya en la ganadería extensiva y la agricultura, actividad que depende de la estacionalidad de las lluvias. El clima es templado seco con estaciones marcadas de lluvia y seca.

Se muestreó la totalidad de la población porcina, con excepción de los animales menores de 2 meses y de las hembras con gestación evidente. Los animales fueron aretados y vacunados contra el cólera porcino (Pestiffa - Merial $\left.{ }^{\circledR}\right)$. El trabajo de campo se realizó en marzo y abril del 2001. 
Cuadro 1. Combinación de bandas, edad y animales positivos a cisticercosis por EITB que fueron empleados para efectuar la simulación estocástica

\begin{tabular}{crrrrr}
\hline \multirow{2}{*}{ Bandas } & \multicolumn{3}{c}{$\leq 8$ meses } & & \multicolumn{2}{c}{$>8$ meses } \\
\cline { 2 - 3 } \cline { 5 - 6 } & + & - & & + & - \\
\hline $1-2$ & 7 & 13 & & 20 & 15 \\
3 & 5 & 7 & & 3 & 1 \\
\hline 4 & 2 & 1 & &
\end{tabular}

Fuente: Dr. Armando González, FMV-UNMSM

+ Positivos

- Negativos

\section{Diagnóstico de cisticercosis}

Se recolectó 8-9 $\mathrm{ml}$ de sangre de la cava anterior en tubos al vacío con gel separador y sin anticoagulante (Vacutainer ${ }^{\circledR}$ ) Se utilizaron los sueros resultantes para determinar la presencia de anticuerpos contra el parásito por medio de la prueba de Electroinmuno Transferencia Blot (EITB).

La prueba tiene un $98 \%$ de sensibilidad y $100 \%$ de especificidad (Tsang y García, 1999; Tsang y Wilson, 1995). El antígeno de EITB fue proporcionado por el Centro de Control de Enfermedades Infecciosas, Atlanta, EE. UU.

Los sueros fueron considerados positivos si al menos estaba presente una de las 7 bandas de glicoproteína (GP) comúnmente reconocidas por anticuerpos de suero de porcinos afectados con cisticercosis: GP 50, GP 42-39, GP 24, GP 21, GP 18, GP 14 y GP 13 ( (Tsang et al., 1989; Tsang et al., 1991).

\section{Análisis estadístico}

Los resultados de seroprevalencia se muestran con intervalos de confianza del $95 \%$. $\mathrm{El}$ efecto de las variables sexo, edad y zona de muestreo sobre la presencia de la enfermedad, expresada como resultado a la prueba serológica de EITB, se evaluó mediante una prueba de regresión logística (Dupont, 2002).
El valor predicho de prevalencia real se estimó con intervalos de confianza del $96 \%$ empleando una simulación beta-binomial usando el paquete @Risk (Palisade Corp.), que por un lado consideró datos de necropsia en la zona andina peruana (A. González, datos no publicados) para fijar distribuciones beta para cada combinación edad/número de bandas, y por otro lado consideró los resultados serológicos observados en cada caserío para predecir el número de cerdos positivos a la necropsia.

Los datos sobre combinaciones de bandas, edad y resultados de necropsia empleados para efectuar la simulación estocástica se presentan en el Cuadro 1. Los datos se obtuvieron luego de efectuar necropsias detalladas en 84 cerdos EITB positivos en un caserío endémico ubicado en el valle del Mantaro (Junín). Los datos se agruparon según combinación de bandas y de acuerdo al límite de 8 meses para la interferencia con anticuerpos maternos.

\section{Resultados}

Se recolectaron muestras de 304 cerdos, resultando 141 animales positivos a cisticercosis, lo que representó una seroprevalencia corregida de $47.3 \pm 5.6 \%$.

La evaluación de las prevalencias de la enfermedad para cada zona indicó que el 
Cuadro 2. Seroprevalencia de cisticercosis porcina, distribuida por caserío, sexo y grupo etáreo. Andahuaylas, Apurímac. 2001

\begin{tabular}{|c|c|c|c|c|}
\hline & & $\begin{array}{c}\text { Animales } \\
\text { muestreados }\end{array}$ & $\begin{array}{l}\text { Animales } \\
\text { positivos }\end{array}$ & $\begin{array}{c}\text { Prevalencia } \\
\text { corregida? IC }{ }^{1}\end{array}$ \\
\hline \multicolumn{5}{|c|}{ Caserío } \\
\hline & Nueva Esperanza & 131 & 34 & $26.5 ? 7.6$ \\
\hline & Turpo & 86 & 61 & $72.3 ? 9.5$ \\
\hline & Matapuquio & 87 & 46 & $54.0 ? 10.5$ \\
\hline \multicolumn{5}{|l|}{ Sexo } \\
\hline & Macho & 149 & 83 & $56.8 ? 8.0$ \\
\hline & Hembra & 155 & 58 & $38.2 ? 7.6$ \\
\hline \multicolumn{5}{|l|}{ Edad } \\
\hline & $>4 \mathrm{~m}$ & 90 & 29 & $32.9 ? 9.7$ \\
\hline & $4-8 \mathrm{~m}$ & 111 & 44 & $40.4 ? 9.1$ \\
\hline & $8-12 \mathrm{~m}$ & 55 & 30 & $55.6 ? 13.1$ \\
\hline & $>12 \mathrm{~m}$ & 42 & 35 & $85.0 ? 10.8$ \\
\hline & Desconocida & 6 & 3 & - - \\
\hline & Total & 304 & 141 & $47.3 ? 5.6$ \\
\hline
\end{tabular}

${ }^{1} \mathrm{IC}=$ Intervalo de confianza al 95\%

caserío de Nueva Esperanza presentó menores niveles de infección respecto a los demás ( $\mathrm{p}<0.05$, Cuadro 2).

Las diferencias entre sexos no fueron significativas, pero se encontró una correlación directa perfecta $\left(\mathrm{r}_{\mathrm{s}}=1\right)$ entre la edad de los animales y la prevalencia de cisticercosis; es decir, que a mayor edad de los animales se tiene una mayor probabilidad de encontrarse infectado (Cuadro 2).

El análisis de regresión logística mostró que las variables zona de muestreo y edad representaron factores de riesgo asociados a la enfermedad ( $<<0.0001)$. En el análisis se consideró el caserío de Nueva Esperanza y a la edad $<4$ meses como estratos de referencia debido a que presentaron la menor proporción de animales infectados (Cuadro 3).

En base a los resultados y luego de correr el modelo de simulación, se encontró que el valor predicho de la prevalencia real para Nueva Esperanza estaría en el intervalo de 7 al 17\%, para Matapuquio entre 19 y $36 \%$ y para Turpo entre 24 y $45 \%$; en tanto que para los tres caseríos sería de $23 \%$ y que el $96 \%$ de las observaciones se encontraría entre el 17 y el $29 \%$. La simulación empleada para calcular la prevalencia real es una solución independiente a la sensibilidad de la prueba. El modelo de simulación ofrece una información mucho más cercana a la realidad en cuanto a la prevalencia de la enfermedad que claramente discrepa de la seroprevalencia, calculada en función de la presencia de anticuerpos.

\section{Discusión}

La prevalencia corregida de cisticercosis porcina para los tres caseríos bajo estudio fue de $47.3 \%$, nivel similar a los hallados en estudios anteriores realizados en la región andina (Ramos, 1999; Bernal, 1996), 
Cuadro 3. Resultado de Odds Ratio a partir de la evaluación del efecto de las variables zona, edad y sexo sobre la presencia de la cisticercosis porcina mediante la prueba de regresión logística

\begin{tabular}{lcccc}
\hline \multirow{2}{*}{ Variable } & $\begin{array}{c}\text { Nivel de } \\
\text { significancia }\end{array}$ & Odds Ratio & \multicolumn{2}{c}{$\mathrm{IC}^{1}$ del 95\% del OR } \\
\cline { 4 - 5 } & & & Límite inferior & Límite superior \\
\hline Zona (Matapuquio) & 0.0003 & 3.25 & 1.71 & 6.22 \\
Zona (Turpo) & 0.0000 & 8.40 & 4.22 & 16.72 \\
Sexo (Macho) & 0.0833 & 1.61 & 0.94 & 2.74 \\
Edad (4-8 meses) & 0.0241 & 2.15 & 1.11 & 4.17 \\
Edad (8-12 meses) & 0.0141 & 2.60 & 1.21 & 5.59 \\
Edad (>12 meses) & 0.0000 & 10.97 & 4.05 & 29.70 \\
\hline
\end{tabular}

${ }^{1}$ Intervalo de confianza

lo que reafirma que esta parasitosis se mantiene como un problema endémico de importancia para la salud pública y la economía del campesino.

El resultado global clasificaría a la provincia de Andahuaylas como zona endémica; sin embargo, al analizar los resultados por caserío se observa que las prevalencias de Turpo (72.3\%) y Matapuquio (54.0\%) son bastante elevadas, lo que indica que estas zonas son hiperendémicas a la enfermedad.

El análisis de regresión logística muestra que la zona de muestreo o caserío representa un factor de riesgo estadísticamente asociado a la enfermedad. Un hecho que podría estar influenciando en los niveles de infección sería la ubicación geográfica y el tipo de vías de acceso. Nueva Esperanza es la más cercana a la capital de la provincia y se llega a través de una carretera afirmada con mucho tránsito, lo que le da la ventaja de un comercio fluido de productos agrícolas y de ganado, de allí que se le da un mayor cuidado e importancia a la crianza de los animales. Los caseríos de Matapuquio y Turpo se encuentran más alejados y con vías de acceso de tipo trocha carrozable, lo que afecta el comercio de productos agrícolas y ganaderos, favoreciendo el descuido de los animales; situación similar a lo encontrado por Ramos (1999) en otros caseríos de esta provincia. La ubicación geográfica de los caseríos afecta en forma diferente el acceso de los pobladores a servicios de salud, educación e información, lo que de alguna forma, no cuantificada hasta el momento, podría además influir en el mantenimiento y dinámica de la enfermedad.

La edad representó un factor de riesgo, hallazgo lógico considerando que el medio ambiente se encuentra altamente contaminado con huevos de Taenia solium, debido a la ausencia de letrinas, y los animales que tienen la oportunidad de vivir más tiempo, tienen más tiempo de exposición. En un deseo de evitar la interferencia de la inmunidad pasiva, se evaluó la edad considerando el límite de la edad como aquel en el que la inmunidad materna tiene una menor influencia ( $>8$ meses), encontrándose que el criterio general no cambiaba.

La importancia de estudios como el presente radica en el hecho de que es necesario monitorear la enfermedad en zonas que 
reúnen condiciones favorables para su presentación, a fin de evaluar el efecto de medidas de control y erradicación del complejo teniasis/cisticercosis. La experiencia indica que los porcinos son los mejores centinelas para medir la infección de este complejo, toda vez que ellos están constantemente muestreando el ambiente (González et al., 1994); a diferencia de hacer evaluaciones en el hombre, donde se encuentran dificultades como el costo de las investigaciones, la baja prevalencia de la teniasis y aspectos culturales, entre otros.

Dada la importancia de esta enfermedad en el país, se debe intensificar los estudios epidemiológicos que permitan conocer y entender a fondo la dinámica de infección de esta parasitosis. Esto permitiría implementar programas de control dirigidos a la detección y tratamiento masivo de poblaciones humanas y porcinas en regiones endémicas.

\section{Literatura Citada}

1. Acha, P.; B. Szyfres. 1992. Zoonosis y enfermedades transmisibles comunes al hombre y a los animales. $2^{\mathrm{a}}$ ed. p 763773. Publicación Científica No 503. OPS.

2. Bernal, T. 1996. Evaluación de la cisticercosis porcina en el distrito de Quilcas, Huancayo. Tesis Bachillerato. Facultad de Medicina Veterinaria, Univ. Nacional Mayor de San Marcos. 45 p.

3. Correa, D.; Z. Morales; Y. Medina; C. García; E. Medina; A. Madujano; D. Ortiz; A Meza. 1991. Teniasis y cisticercosis por Taenia solium. Publicación Técnica del INDRE. No 4. p 1-45.

4. Dupont, W.D. 2002. Statistical modeling for biomedical research. A simple introduction to the analysis of complex data. p 108-226. Cambridge University Press.

4. Evans, C.; H. García; R. Gilman. 2000. Cysticercosis. En: Hunter's tropical medicine and emerging infectious diseases. $8^{\text {th }}$ ed. W.B. Sander Co., Phyladelphia.

5. García, H.H.; M. Martínez; R. Gilman; G. Herrera; V. Tsang; J. Pilcher; F. Díaz; M. Verástegui; $C$. Gallo; M. Porras; M. Alvarado; J. Naranjo; E. Miranda; The Cysticercosis working Group in Peru (CWG). 1991. Diagnosis of cysticer-cosis in endemic regions. The Lancet 338: 549351.

6. García, H.H.; R. Gilman; M. Tovar; E. Flores; R. Jo; V. Tsang; F. Díaz; P. Torres; E. Miranda; The Cysticercosis Working Group in Peru (CWG). 1995. Factors associated with Taenia solium cysticercosis: analysis of nine hundred forty six Peruvian neurologic patients. Am. J. Med. Hyg. 52: 145-148.

7. García, H.H.; R. Gilman; A.E. González; M. Verástegui; The Cysticercosis Working Group in Peru (CWG). 1999a. Epidemiology of Taenia solium infection in Peru. En: Taeniasis/ Cisticercosis by Taenia solium. Section

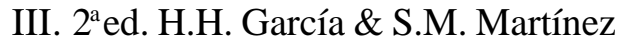
(eds). Ed. Universo. Lima. Perú.

8. García, H.H.; R. Gilman; A.E. González; R. Pacheco; M. Verástegui; $V$. Tsang; The Cysticercosis Working Group in Peru (CWG). 1999b. Human and porcine Taenia solium infection in a village in the highlands of Cusco, Peru. Acta Tropical 73: 31-36.

9. González, A.E.; V. Cama; R. Gilman; V. Tsang; J. Pilcher; A. Chavera; M. Castro; T. Montenegro; M. Verástegui; E. Miranda; E. Bazalar. 1990. Prevalence and comparison of serologic assay, necropsy and tongue examination for the diagnosis of porcine cisticercosis in Peru. Am. J. Trop. Med. Hyg. 43: 194199.

10. González, A.E.; R. Gilman; H.H. García; J. McDonald; K. Kacena; V. Tsang; J. Pilcher; F. Suárez; C. Gavidia; E. Miranda; The Cysticercosis Working Group in Peru (CWG). 1994. Use of sentinel pigs to monitor 
environmental Taenia solium contamination. Am. J. Med. Hyg. 51: 847-850.

11. Organización Panamericana de la Salud / Organización Mundial de la Salud. 1994. Epidemiología y control de la teniasis/cisticercosis en América Latina. Versión 3.0 REF: PNSP/91-28.

12. Ramos, D. 1999. Seroprevalencia de cisticercosis porcina en las villas de Occollo y Anaccma - Provincia de Andahuaylas-Departamento de Apurímac. Tesis Bachillerato. Facultad de Medicina Veterinaria, Univ. Nacional Mayor de San Marcos. Lima. $51 \mathrm{p}$.

13. The Cysticercosis Working Group in Peru. 1993. The marketing of cysticercotic pigs in the sierra of Peru. Bull. World Health Organization 71: 223-228.

14. Tsang, V.C.W.; J. Brand; A. Boyer. 1989. An enzyme-linked immunoelectrotransfer blot assay and glycoprotein antigens for diagnosing human cysticercosis (Taenia solium). J. Infec. Dis. 159: 50-59.
15. Tsang, V.C.W.; J. Brand; W. Zhou; A. Boyer; E. Kamango-Sollo; M. Rhoads; K. Darwin; P. Schantz; R. Gilman. 1991. Efficacy of the immunobloth assay for cysticercosis in pigs and modulated expression of distinct $\operatorname{Ig} \mathrm{M} / \mathrm{Ig} \mathrm{G}$ activities to Taenia solium antigens in experimental infections. Vet. Immunology and Immunopathology 29: 69-78.

16. Tsang, V.C.W.; M. Wilson. 1995. Taenia solium cysticercosis: an underrecognized but serius public health problem. Parasitology Today 11: 124-126.

17. Tsang, V.; H.H. García. 1999. Inmunoblot diagnostic test (EITB) for Taenia solium cysticercosis and its contribution to the definition of this underrecognized but serius public health problem. En: Taeniasis/Cisticercosis by Taenia solium. Section III. $2^{a}$ ed. H.H. García \& S.M. Martínez (eds.) Ed. Universo. Lima. Perú. 